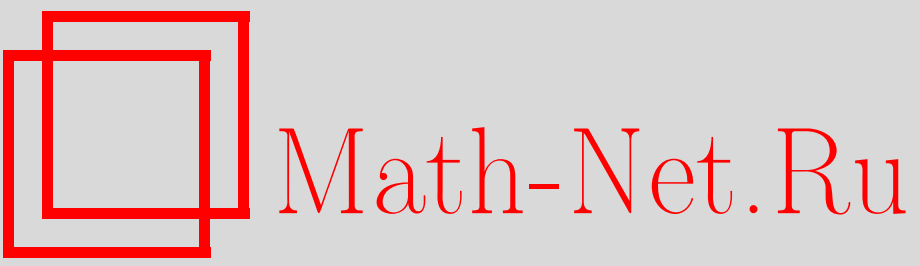

В. В. Жаринов, Когомологии алгебры Ли векторных полей на прямой, ТМФ, 2001, том 128, номер 2, 147-160

DOI: https://doi.org/10.4213/tmf488

Использование Общероссийского математического портала Math-Net.Ru подразумевает, что вы прочитали и согласны с пользовательским соглашением

http://www.mathnet.ru/rus/agreement

Параметры загрузки:

IP : 54.197 .130 .99

26 апреля 2023 г., 13:52:09 
ТЕОРЕТИЧЕСКАЯ

И МАТЕМАТИЧЕСКАЯ

ФИЗИКА

Том 128, № 2

август, 2001

(C) 2001 г.

В.В. Жаринов

\section{КОГОМОЛОГИИ АЛГЕБРЫ ЛИ ВЕКТОРНЫХ ПОЛЕЙ НА ПРЯМОЙ}

Предложен адекватный математический аппарат и найдены когомологии алгебры Ли гладких векторных полей на прямой с коэффициентами в важнейших представлениях, а именно: в тривиальном, естественном и присоединенном. Построена обобщающая серия комплексов и вычислены соответствующие когомологии.

Современное развитие теоретической и как следствие математической физики происходит под знаком их глубокой и всесторонней геометризации и алгебраизации. Глядя со стороны, можно подумать, что сейчас математическая физика есть своеобразная смесь специфических разделов геометрии и алгебры с известной долей анализа и теории уравнений в частных производных. Одну из центральных ролей в этом ансамбле играют алгебры Ли, причем следует отметить, что хотя вся история классической и квантовой физики неразрывно связана с симметриями и их математическим выражением группами и алгебрами Ли, в настояшее время совершается переход от конечномерных алгебр Ли, связанных с симметриями в физическом и изотопических пространствах, к бесконечномерным алгебрам, характерным для новейших квантовых теорий, например для теории струн и калибровочных моделей. Внедряясь в физику, математические конструкции привносят в нее присущие им объекты и методы исследования, давая единую базу для описания первоначально разнородных, не связанных между собой физических явлений. Один из многочисленных примеров - классификация элементарных частиц на основе представлений групп Ли.

Естественным языком, описывающим многие объекты, связанные с алгебрами Ли и их представлениями, являются когомологии алгебр Ли [1], которые одновременно дают адекватные методы исследования, классификации и нахождения естественных связей между изучаемыми физическими величинами. Особенно эффективным такой подход становится в бесконечномерном случае [2], [3], когда классические методы становятся неприменимыми. О современных тенденциях использования когомологий алгебр Ли в физике можно прочитать, например, в [4] (см. также цитируемую там литературу). В общем, судя по количеству публикаций в электронных архивах и физических журналах, можно заключить, что когомологии алгебр Ли все шире вовлекаются в теоретическую

* Математический институт им. В. А. Стеклова РАН, Москва, Россия. E-mail: victor@zharinov.mian.su; zharinov@mi.ras.ru 
и математическую физику.

Важными для приложений элементами теории когомологий алгебр Ли являются вычислительный аппарат и набор полностью просчитанных характерных примеров. Известные результаты в этой области описаны в книге [2] и обзоре [3], где имеются многочисленные ссылки на оригинальные работы. Возможно, важнейшим классом бесконечномерных алгебр Ли являются алгебры Ли векторных полей на многообразиях. Простейшие из них - алгебры на единичной окружности и на прямой. Первые, как и вообще алгебры полей на компактных многообразиях, изучены лучше, поскольку в этом случае доступна техника инвариантного интегрирования [5] (см. [2], [3] и приведенные там ссылки). Наиболее полные результаты получены в случае коэффициентов в тривиальном представлении.

В предлагаемой работе вычисляются когомологии алгебры Ли гладких векторных полей на прямой с коэффициентами в трех важнейших представлениях, а именно: в тривиальном, естественном и присоединенном. Попутно разработан адекватный математический аппарат, сводяший вычисления к элементарным выкладкам и позволяюший легко обобшать рассмотренньй случай. Основной упор сделан на алгебраическую, комбинаторную часть рассуждений, как на наиболее характерную для изучаемого вопроса, при этом аналитическая часть оставлена в стороне, как более тривиальная, не содержашая чего-либо необычного.

\section{1. ОПРЕДЕЛЕНИЯ И ФОРМУЛИРОВКИ РЕЗУЛЬТАТОВ}

1.1. Когомологии алгебр Ли. Пусть $\mathbb{F}$ - поле характеристики нуль, $\mathfrak{A}$ - алгебра Ли над $\mathbb{F}$ со скобкой $[\cdot, \cdot], \mathfrak{K}$ - модуль над $\mathfrak{A}$, т.е. $\mathfrak{K}$ есть линейное пространство над $\mathbb{F}$, и задано представление $\rho$ алгебры Ли $\mathfrak{A}$ в $\mathfrak{K}$,

$$
\rho: \mathfrak{A} \rightarrow \operatorname{Hom}_{\mathbb{F}}(\mathfrak{K} ; \mathfrak{K}), \quad a \mapsto \rho(a): \mathfrak{K} \rightarrow \mathfrak{K} \quad \forall a \in \mathfrak{A},
$$

так что, в частности, выполняется коммутаторное правило

$$
\rho([a, b])=[\rho(a), \rho(b)] \equiv \rho(a) \circ \rho(b)-\rho(b) \circ \rho(a) \quad \forall a, b \in \mathfrak{A},
$$

где "о" - знак композиции отображений.

Пусть

$$
\wedge \mathfrak{A}=\bigoplus_{q \geqslant 0} \wedge^{q} \mathfrak{A}
$$

- внешняя алгебра линейного пространства $\mathfrak{A}$ над $\mathbb{F}$,

$$
\mathcal{C}(\mathfrak{A} ; \mathfrak{K})=\bigoplus_{q \geqslant 0} \mathcal{C}^{q}(\mathfrak{A} ; \mathfrak{K})
$$


- линейное пространство над $\mathbb{F}$ всех коцепей на $\mathfrak{A}$ со значениями в $\mathfrak{K}$, где $\mathcal{C}^{q}(\mathfrak{A} ; \mathfrak{K})=$ $\operatorname{Hom}_{\mathbb{F}}(\wedge q \mathfrak{A} ; \mathfrak{K})$ - линейные пространства всех $q$-линейных кососимметрических отображений из $\mathfrak{A}$ в $\mathfrak{K}$. Внешний дифференциал $d$ на $\mathcal{C}(\mathfrak{A} ; \mathfrak{K})$ задается правилом

$$
\begin{aligned}
(d \omega)\left(a_{0}, \ldots, a_{q}\right)= & \frac{1}{q+1}\left\{\sum_{0 \leqslant \alpha \leqslant q}(-1)^{\alpha} \rho\left(a_{\alpha}\right)\left(\omega\left(a_{0}, \ldots, \check{a_{\alpha}}, \ldots, a_{q}\right)\right)+\right. \\
& \left.+\sum_{0 \leqslant \alpha<\beta \leqslant q}(-1)^{\alpha+\beta} \omega\left(\left[a_{\alpha}, a_{\beta}\right], \ldots, \check{a_{\alpha}}, \ldots, \check{a_{\beta}}, \ldots, a_{q}\right)\right\}
\end{aligned}
$$

для $\omega \in \mathcal{C}^{q}(\mathfrak{A} ; \mathfrak{K})$ и $a_{0}, \ldots, a_{q} \in \mathfrak{A}$ (аргумент под значком " $\sim$ " опускается), так что $d$ : $\mathcal{C}^{q}(\mathfrak{A} ; \mathfrak{K}) \rightarrow \mathcal{C}^{q+1}(\mathfrak{A} ; \mathfrak{K}), q \in \mathbb{Z}_{+}=\{0,1, \ldots\}$. В силу тождества Якоби и коммутаторного правила композиция $d \circ d=0$, так что определен комплекс $\{\mathcal{C}(\mathfrak{A} ; \mathfrak{K}) ; d\}$ с когомологиями

$$
H(\{\mathcal{C}(\mathfrak{A} ; \mathfrak{K}) ; d\})=H(\mathfrak{A} ; \mathfrak{K})=\bigoplus_{q \geqslant 0} H^{q}(\mathfrak{A} ; \mathfrak{K}) .
$$

1.2. Алгебра Ли гладких векторных полей на прямой. Пусть $\mathbb{F}=\mathbb{C}, \mathcal{E}$ - ассоциативная коммутативная алгебра над $\mathbb{C}$ всех гладких (т.е. имеюших непрерывные производные всех порядков) комплекснозначных функций на прямой $\mathbb{R}$ с обычными поточечными операциями, $\mathfrak{A}$ - алгебра Ли всех гладких векторных полей на прямой (т.е. всех гладких дифференцирований алгебры $\mathcal{E}$ ). Подробнее,

$$
\mathfrak{A}=\left\{u=u(x) \frac{d}{d x} ; \quad u(x) \in \mathcal{E}, \quad x \in \mathbb{R}\right\},
$$

скобка Ли $[u, v]=w$, где $w=w(x) d / d x$,

$$
w(x)=u(x) v^{\prime}(x)-v(x) u^{\prime}(x)=W_{2}[u, v](x)
$$

есть вронскиан двух функций $u(x), v(x) \in \mathcal{E}$, вычисленный в точке $x \in \mathbb{R}$ (штрих означает производную по $x \in \mathbb{R}$ ). В частности, наделив линейное пространство $\mathcal{E}$ скобкой Ли, определяемой вронскианом, отождествим алгебры Ли $\mathfrak{A}$ и $\mathcal{E}$.

Важнейшие представления алгебры Ли $\mathfrak{A}$ суть:

а) тривиальное представление, где $\mathfrak{K}=\mathbb{C}$ и $\rho(u)=0, u \in \mathfrak{A}$;

б) естественное представление, где $\mathfrak{K}=\mathcal{E}$ и $\rho(u)=u(x) d / d x, u \in \mathfrak{A}$;

в) присоединенное представление, где $\mathfrak{K}=\mathfrak{A}$ и $\rho(u)=[u, \cdot], u \in \mathfrak{A}$.

Отметим, что тривиальное и присоединенное представления есть у любой алгебры Ли, а естественное - у любой алгебры Ли дифференцирований ассоциативной алгебры.

1.3. Когомологии, формулировки результатов. В случае коцепей с коэффициентами в тривиальном представлении внешний дифференциал действует по формуле

$$
(d \omega)\left(u_{0}, \ldots, u_{q}\right)=\frac{1}{q+1} \sum_{0 \leqslant \alpha<\beta \leqslant q}(-1)^{\alpha+\beta} \omega\left(\left[u_{\alpha}, u_{\beta}\right], u_{0}, \ldots, \check{u_{\alpha}}, \ldots, \check{u_{\beta}}, \ldots, u_{q}\right)
$$

для всех $\omega \in \mathcal{C}^{q}(\mathfrak{A} ; \mathbb{C}), u_{0}, \ldots, u_{q} \in \mathfrak{A}$ и $q \in \mathbb{Z}_{+}$. 
ТЕОрема 1. Когомологии алгебры Ли $\mathfrak{A}$ гладких векторных полей на прямой с коэффициентами в тривиальном представлении, т.е. когомологии комплекса $\{\mathcal{C}(\mathfrak{A} ; \mathbb{C}) ; d\}$, равны

$$
H^{q}(\mathfrak{A} ; \mathbb{C})= \begin{cases}\mathbb{C}, & q=0, \\ \mathbb{C} \cdot W_{3}(0), & q=3 \\ 0, & q \neq 0,3\end{cases}
$$

где $W_{3}[u, v, w](0)$ - вронскиан трех функций $u, v, w \in \mathcal{E}$, вычисленный в точке $0 \in \mathbb{R}$.

В случае коцепей с коэффициентами в естественном представлении внешний дифференциал действует по формуле

$$
\begin{aligned}
(d \omega)\left(x ; u_{0}, \ldots, u_{q}\right)= & \frac{1}{q+1}\left\{\sum_{0 \leqslant \alpha \leqslant q}(-1)^{\alpha} u_{\alpha}(x)\left(\omega\left(x ; u_{0}, \ldots, \check{u_{\alpha}}, \ldots, u_{q}\right)\right)_{x}^{\prime}+\right. \\
& \left.+\sum_{0 \leqslant \alpha<\beta \leqslant q}(-1)^{\alpha+\beta} \omega\left(x ;\left[u_{\alpha}, u_{\beta}\right], u_{0}, \ldots, \check{u_{\alpha}}, \ldots, \check{u_{\beta}}, \ldots, u_{q}\right)\right\}
\end{aligned}
$$

для всех $\omega \in \mathcal{C}^{q}(\mathfrak{A} ; \mathcal{E}), u_{0}, \ldots, u_{q} \in \mathfrak{A}$ и $q \in \mathbb{Z}_{+}$.

ТЕОРема 2. Когомологии алгебры Ли $\mathfrak{A}$ гладких векторных полей на прямой с коэффичиентами в естественном представлении, т.е. когомологии комплекса $\{\mathcal{C}(\mathfrak{A} ; \mathcal{E}) ; d\}$, равны

$$
H^{q}(\mathfrak{A} ; \mathcal{E})= \begin{cases}\mathbb{C}, & q=0, \\ \mathbb{C} \cdot \frac{d}{d x}, & q=1, \\ 0, & q \geqslant 2 .\end{cases}
$$

В случае коцепей с коэффициентами в присоединенном представлении внешний дифференциал действует по формуле

$$
\begin{aligned}
(d \omega)\left(x ; u_{0}, \ldots, u_{q}\right)= & \frac{1}{q+1}\left\{\sum_{0 \leqslant \alpha \leqslant q}(-1)^{\alpha} W_{2}\left[u_{\alpha}, \omega\left(u_{0}, \ldots, \check{u_{\alpha}}, \ldots, u_{q}\right)\right](x)+\right. \\
& \left.+\sum_{0 \leqslant \alpha<\beta \leqslant q}(-1)^{\alpha+\beta} \omega\left(x ;\left[u_{\alpha}, u_{\beta}\right], u_{0}, \ldots, \check{u_{\alpha}}, \ldots, \check{u_{\beta}}, \ldots, u_{q}\right)\right\}
\end{aligned}
$$

для всех $\omega \in \mathcal{C}^{q}(\mathfrak{A} ; \mathfrak{A}), u_{0}, \ldots, u_{q} \in \mathfrak{A}$ и $q \in \mathbb{Z}_{+}$.

ТЕОРема 3. Когомологии алгебры Ли А гладких векторных полей на прямой с коэффициентами в присоединенном представлении, т.е. когомологии комплекса $\{\mathcal{C}(\mathfrak{A} ; \mathfrak{A}) ; d\}$, тривиальнье, подробнее

$$
H^{q}(\mathfrak{A} ; \mathfrak{A})=0, \quad q \in \mathbb{Z}_{+} .
$$

Остальная часть статьи посвяшена доказательству перечисленных теорем и разработке адекватного математического аппарата. 


\section{2. МАТЕМАТИЧЕСКИЙ АППАРАТ}

2.1. Экспоненциальное преобразование. Каждому $\xi \in \mathbb{C}$ поставим в соответствие функцию $e^{\xi} \in \mathcal{E}$, где $e^{\xi}(x)=e^{x \xi}, x \in \mathbb{R}$, причем $\left[e^{\xi}, e^{\eta}\right]=(\eta-\xi) e^{\xi+\eta}$ для всех $\xi, \eta \in \mathbb{C}$.

Приведем без излишних подробностей ряд определений и результатов из теории обобщенных функций, используемых в дальнейшем. Пусть $\mathcal{E}\left(\mathbb{R}^{q}\right)$ - линейное локально-выпуклое пространство всех гладких функций на $\mathbb{R}^{q}$ с топологией равномерной сходимости на компактах в $\mathbb{R}^{q}$ вместе с частными производными любого порядка. Его сильное сопряженное пространство $\mathcal{E}^{\prime}\left(\mathbb{R}^{q}\right)$ есть линейное локально-выпуклое пространство всех обобщенных функций с компактным носителем на $\mathbb{R}^{q}$ с топологией равномерной сходимости на ограниченных множествах из $\mathcal{E}\left(\mathbb{R}^{q}\right)$. Пусть $\operatorname{Exp}\left(\mathbb{C}^{q}\right)$ - линейное пространство всех целых функций на $\mathbb{C}^{q}$ экспоненциального типа. Преобразование Лапласа $L: \mathcal{E}^{\prime}\left(\mathbb{R}^{q}\right) \rightarrow \operatorname{Exp}\left(\mathbb{C}^{q}\right), \omega \mapsto L[\omega]$, задается формулой $L[\omega]\left(\xi_{1}, \ldots, \xi_{q}\right)=\omega\left(e^{\xi_{1}}, \ldots, e^{\xi_{q}}\right)$ для всех $\omega \in \mathcal{E}^{\prime}\left(\mathbb{R}^{q}\right)$ и $\xi=\left(\xi_{1}, \ldots, \xi_{q}\right) \in \mathbb{C}^{q}$. Линейное отображение $L$ инъективно, его образ $L\left[\mathcal{E}^{\prime}\left(\mathbb{R}^{q}\right)\right] \subset \operatorname{Exp}\left(\mathbb{C}^{q}\right)$ состоит из всех целых функций на $\mathbb{C}^{q}$, растущих не быстрее линейной экспоненты в вешественных направлениях и не быстрее степени в мнимых направлениях. Линейное пространство $L\left[\mathcal{E}^{\prime}\left(\mathbb{R}^{q}\right)\right]$ наделяется локально-выпуклой топологией, индуцированной из $\mathcal{E}^{\prime}\left(\mathbb{R}^{q}\right)$, так что определен изоморфизм линейных локально-выпуклых пространств $L: \mathcal{E}^{\prime}\left(\mathbb{R}^{q}\right) \simeq L\left[\mathcal{E}^{\prime}\left(\mathbb{R}^{q}\right)\right]$ (см., например, [6]).

По построению линейное пространство $q$-коцепей $\mathcal{C} q(\mathfrak{A} ; \mathbb{C})$ есть подпространство в $\mathcal{E}^{\prime}\left(\mathbb{R}^{q}\right)$, состоящее из всех кососимметрических обобщенных функций с компактным носителем на $\mathbb{R}^{q}$, подробнее

$$
\mathcal{C}^{q}(\mathfrak{A} ; \mathbb{C})=\left\{\omega \in \mathcal{E}^{\prime}\left(\mathbb{R}^{q}\right): \pi^{*} \omega=\operatorname{sign}(\pi) \omega \text { для всех } \pi \in \Sigma_{q}\right\},
$$

где $\Sigma_{q}$ - множество всех перестановок индексов $\{1, \ldots, q\}, \operatorname{sign}(\pi)$ - знак перестановки $\pi \in \Sigma_{q},\left(\pi^{*} \omega\right)\left(u_{1}, \ldots, u_{q}\right)=\omega\left(u_{\pi(1)}, \ldots, u_{\pi(q)}\right)$. Наделяя образ $\Phi^{q}=L\left[\mathcal{C}^{q}(\mathfrak{A} ; \mathbb{C})\right] \subset$ $L\left[\mathcal{E}^{\prime}\left(\mathbb{R}^{q}\right)\right]$ локально-выпуклой топологией, индуцированной из $L\left[\mathcal{E}^{\prime}\left(\mathbb{R}^{q}\right)\right]$, получаем изоморфизм линейных пространств $L: \mathcal{C}^{q}(\mathfrak{A} ; \mathbb{C}) \simeq \Phi^{q}$. Заметим, что $\mathcal{C}^{0}(\mathfrak{A} ; \mathbb{C})=\Phi^{0}=\mathbb{C}$. Для $q=1,2, \ldots$ линейное локально-выпуклое пространство $\Phi^{q}$ состоит из всех целых кососимметрических функций на $\mathbb{C}^{q}$, растуших не быстрее линейной экспоненты в вешественных направлениях и не быстрее степени в мнимых направлениях, и наделено топологией, индуцированной из $\mathcal{E}^{\prime}\left(\mathbb{R}^{q}\right)$. Итак, определен изоморфизм $L: \mathcal{C}(\mathfrak{A} ; \mathbb{C}) \simeq \Phi$, где $\Phi=\bigoplus_{q \geqslant 0} \Phi^{q}$.

Далее, линейное пространство $q$-коцепей $\mathcal{C}^{q}(\mathfrak{A} ; \mathcal{E})=\mathcal{E} \widehat{\otimes} \mathcal{C}^{q}(\mathfrak{A} ; \mathbb{C})$, где символ $\widehat{\otimes}$ означает замыкание тензорного произведения в подходяшей топологии, которая для нас сейчас не важна (заметим, что $\mathcal{E}=\mathcal{E}\left(\mathbb{R}^{1}\right)$ ). Отождествление $L=\mathrm{id}_{\mathcal{E}} \otimes L$ распространяет преобразование Лапласа на $\mathcal{C}^{q}(\mathfrak{A} ; \mathcal{E})$ и приводит к изоморфизму $L: \mathcal{C}^{q}(\mathfrak{A} ; \mathcal{E}) \simeq \mathcal{F}^{q}$, где $\mathcal{F}^{q}=\mathcal{E} \widehat{\otimes} \Phi^{q}$. Введем "подкрученное" преобразование $\Lambda: \mathcal{C}^{q}(\mathfrak{A} ; \mathcal{E}) \simeq \mathcal{F}^{q}$, где

$$
\Lambda[\omega]\left(x ; \xi_{1}, \ldots, \xi_{q}\right)=\exp \left(-x \sum_{1 \leqslant \alpha \leqslant q} \xi_{\alpha}\right) L[\omega]\left(x ; \xi_{1}, \ldots, \xi_{q}\right)
$$


152

В.В. ЖАРИНОВ

для всех $\omega \in \mathcal{C}^{q}(\mathfrak{A} ; \mathcal{E}), \quad x \in \mathbb{R}, \xi=\left(\xi_{1}, \ldots, \xi_{q}\right) \in \mathbb{C}^{q}$. Итак, определен изоморфизм линейных пространств $\Lambda: \mathcal{C}(\mathfrak{A} ; \mathcal{E}) \simeq \mathcal{F}$, где $\mathcal{F}=\bigoplus_{q \geqslant 0} \mathcal{F}^{q}, \mathcal{C}^{0}(\mathfrak{A} ; \mathcal{E})=\mathcal{F}^{0}=\mathcal{E}$.

Как было указано, линейные пространства $\mathfrak{A}$ и $\mathcal{E}$ совпадают, так что определен и изоморфизм линейных пространств $\Lambda: \mathcal{C}(\mathfrak{A} ; \mathfrak{A}) \simeq \mathcal{F}$.

ПРЕДЛОЖЕНИЕ 1. Имеется изоморфизм комплексов

$$
L:\{\mathcal{C}(\mathfrak{A} ; \mathbb{C}) ; d\} \simeq\left\{\Phi ; d_{\mathbb{C}}\right\}
$$

где дифференциал $d_{\mathbb{C}}=L \circ d \circ L^{-1}: \Phi \rightarrow \Phi$ действует по формуле

$$
\begin{aligned}
&\left(d_{\mathbb{C}} \phi\right)\left(\xi_{0}, \ldots, \xi_{q}\right)= \\
&=\frac{1}{q+1} \sum_{0 \leqslant \alpha<\beta \leqslant q}(-1)^{\alpha+\beta-1}\left(\xi_{\alpha}-\xi_{\beta}\right) \phi\left(\xi_{\alpha}+\xi_{\beta}, \xi_{0}, \ldots, \check{\xi_{\alpha}}, \ldots, \check{\xi_{\beta}}, \ldots, \xi_{q}\right) \\
& \text { для всех } \phi \in \Phi^{q}, \quad \xi_{0}, \ldots, \xi_{q} \in \mathbb{C}, \quad q \in \mathbb{Z}_{+} .
\end{aligned}
$$

ПРЕДЛОЖЕНИЕ 2. Имеется изоморфизм комплексов

$$
\Lambda:\{\mathcal{C}(\mathfrak{A} ; \mathcal{E}) ; d\} \simeq\left\{\mathcal{F} ; d_{\mathcal{E}}\right\}
$$

где дифференциал $d_{\mathcal{E}}=\Lambda \circ d \circ \Lambda^{-1}: \mathcal{F} \rightarrow \mathcal{F}$ действует по формуле

$$
\begin{aligned}
& \left(d_{\mathcal{E}} f\right)\left(x ; \xi_{0}, \ldots, \xi_{q}\right)= \\
& =\frac{1}{q+1}\left\{\sum_{0 \leqslant \alpha \leqslant q}(-1)^{\alpha}\left(\partial_{x}+\sum_{0 \leqslant \beta \leqslant q} \xi_{\beta}-\xi_{\alpha}\right) f\left(x ; \xi_{0}, \ldots, \check{\xi_{\alpha}}, \ldots, \xi_{q}\right)+\right. \\
& \left.\quad+\sum_{0 \leqslant \alpha<\beta \leqslant q}(-1)^{\alpha+\beta-1}\left(\xi_{\alpha}-\xi_{\beta}\right) f\left(x ; \xi_{\alpha}+\xi_{\beta}, \xi_{0}, \ldots, \check{\xi}_{\alpha}, \ldots, \check{\xi_{\beta}}, \ldots, \xi_{q}\right)\right\} \\
& \text { для всех } f \in \mathcal{F}^{q}, \quad \xi_{0}, \ldots, \xi_{q} \in \mathbb{C}, q \in \mathbb{Z}_{+} .
\end{aligned}
$$

ПРЕДЛОЖЕНИЕ 3. Имеется изоморфизм комплексов

$$
\Lambda:\{\mathcal{C}(\mathfrak{A} ; \mathfrak{A}) ; d\} \simeq\left\{\mathcal{F} ; d_{\mathfrak{A}}\right\},
$$

где дифференчиал $d_{\mathfrak{A}}=\Lambda \circ d \circ \Lambda^{-1}: \mathcal{F} \rightarrow \mathcal{F}$ действует по формуле

$$
\begin{aligned}
&\left(d_{\mathfrak{A}} f\right)\left(x ; \xi_{0}, \ldots, \xi_{q}\right)= \\
&= \frac{1}{q+1}\left\{\sum_{0 \leqslant \alpha \leqslant q}(-1)^{\alpha}\left(\partial_{x}+\sum_{0 \leqslant \beta \leqslant q} \xi_{\beta}-2 \xi_{\alpha}\right) f\left(x ; \xi_{0}, \ldots, \check{\xi_{\alpha}}, \ldots, \xi_{q}\right)+\right. \\
&\left.\quad+\sum_{0 \leqslant \alpha<\beta \leqslant q}(-1)^{\alpha+\beta-1}\left(\xi_{\alpha}-\xi_{\beta}\right) f\left(x ; \xi_{\alpha}+\xi_{\beta}, \xi_{0}, \ldots, \check{\xi_{\alpha}}, \ldots, \check{\xi_{\beta}}, \ldots, \xi_{q}\right)\right\}
\end{aligned}
$$

для всех $f \in \mathcal{F}^{q}, \xi_{0}, \ldots, \xi_{q} \in \mathbb{C}, q \in \mathbb{Z}_{+}$.

Доказательства предложений 1-3 проводятся элементарными вычислениями. 
2.2. Операции в пространстве $\Phi$. В пространствах $\Phi^{q}, q \in \mathbb{Z}_{+}$, выделим подпространства

$\Phi^{p, q}=\left\{\phi(\xi)=\sum_{|n|=p} \phi_{n} \xi^{n} \in \Phi^{q}\right\}$ однородных многочленов,

$\Phi_{\neq p}^{q}=\left\{\phi(\xi)=\sum_{|n| \neq p} \phi_{n} \xi^{n} \in \Phi^{q}\right\}$ их дополнений и

$\Phi_{\geqslant p}^{q}=\left\{\phi(\xi)=\sum_{|n| \geqslant p} \phi_{n} \xi^{n} \in \Phi^{q}\right\}$ остатков,

где порядок $p \in \mathbb{Z}_{+}$, аргумент $\xi=\left(\xi_{1}, \ldots, \xi_{q}\right) \in \mathbb{C}^{q}$, мультииндекс $n=\left(n_{1}, \ldots, n_{q}\right) \in$ $\mathbb{Z}_{+}^{q}$, его модуль $|n|=\sum_{1 \leqslant \alpha \leqslant q} n_{\alpha}$, коэффиициент $\phi_{n} \in \mathbb{C}$, моном $\xi^{n}=\xi_{1}^{n_{1}} \cdots \xi_{q}^{n_{q}}$.

По построению функции $\phi \in \Phi^{q}, q \in \mathbb{Z}_{+}$, кососимметрические, т.е. $\pi^{*} \phi=\operatorname{sign}(\pi) \phi$ для всех $\pi \in \Sigma_{q}$, где $\left(\pi^{*} \phi\right)\left(\xi_{1}, \ldots, \xi_{q}\right)=\phi\left(\xi_{\pi(1)}, \ldots, \xi_{\pi(q)}\right), \xi_{1}, \ldots, \xi_{q} \in \mathbb{C}$. Это приводит к следуюшему утверждению.

ПРЕДЛОЖЕНИЕ 4. Пусть $\phi \in \Phi^{q}, \quad q \in \mathbb{Z}_{+}$, тогда $\phi(\xi)=V_{q}(\xi) \cdot \rho(\xi)$, əде

$$
V_{q}(\xi)=\prod_{1 \leqslant \alpha<\beta \leqslant q}\left(\xi_{\beta}-\xi_{\alpha}\right)
$$

- определитель Вандермонда, $\rho(\xi)$ - симметрическая иелая функиия с оченками того жее вида, что и $\phi(\xi), \xi=\left(\xi_{1}, \ldots, \xi_{q}\right) \in \mathbb{C}^{q}$.

В частности, $\Phi^{p, q}=0$ при $p<\operatorname{deg} V_{q}=q(q-1) / 2$.

Заметим, что $L^{-1}\left[V_{q}\right]=W_{q}[\cdots](0)$ - вронскиан, вычисленный в точке $0 \in \mathbb{R}$, а $\Lambda^{-1}\left[V_{q}\right](x)=W_{q}[\cdots](x)$ - вронскиан, вычисленный в точке $x \in \mathbb{R}$

Введем на пространстве $\Phi$ ряд операций.

- Линейные отображения $\nabla_{l}^{k}: \Phi \rightarrow \Phi, k, l \in \mathbb{Z}_{+}$, зададим формулой

$$
\left(\nabla_{l}^{k} \phi\right)\left(\xi_{1}, \ldots, \xi_{q}\right)=\frac{1}{l !} \sum_{1 \leqslant \alpha \leqslant q} \xi_{\alpha}^{k} \partial_{\alpha}^{l} \phi\left(\xi_{1}, \ldots, \xi_{q}\right)
$$

для всех $\phi \in \Phi^{q}, \xi_{1}, \ldots, \xi_{q} \in \mathbb{C}$, где частные производные $\partial_{\alpha}=\partial / \partial \xi_{\alpha}$. В частности, $\nabla_{l}^{k}: \Phi^{p, q} \rightarrow \Phi^{p+k-l, q}$, причем

$$
\left.\nabla_{0}^{0}\right|_{\Phi^{p, q}}=q \operatorname{id}_{\Phi, q},\left.\quad \nabla_{1}^{1}\right|_{\Phi^{p, q}}=p \operatorname{id}_{\Phi, q}
$$

для всех $p, q \in \mathbb{Z}_{+}$.

- Линейные отображения $\lambda^{k}: \Phi \rightarrow \Phi, k \in \mathbb{Z}_{+}$, зададим формулой

$$
\left(\lambda^{k} \phi\right)\left(\xi_{0}, \ldots, \xi_{q}\right)=\frac{1}{q+1} \sum_{0 \leqslant \alpha \leqslant q}(-1)^{\alpha} \xi_{\alpha}^{k} \phi\left(\xi_{0}, \ldots, \check{\xi_{\alpha}}, \ldots, \xi_{q}\right)
$$

для всех $\phi \in \Phi^{q}, \xi_{0}, \ldots, \xi_{q} \in \mathbb{C}$. В частности, $\lambda^{k}: \Phi^{p, q} \rightarrow \Phi^{p+k, q+1}$ для всех $p, q \in \mathbb{Z}_{+}$.

- Линейные отображения $\iota_{k}: \Phi \rightarrow \Phi, k \in \mathbb{Z}_{+}$, зададим формулой

$$
\left(\iota_{k} \phi\right)\left(\xi_{1}, \ldots, \xi_{q-1}\right)=\left.\frac{q}{k !}\left(\partial_{0}^{k} \phi\left(\xi_{0}, \xi_{1}, \ldots, \xi_{q-1}\right)\right)\right|_{\xi_{0}=0}
$$

для всех $\phi \in \Phi^{q}, \xi_{1}, \ldots, \xi_{q-1} \in \mathbb{C}$. В частности, $\iota_{k}: \Phi^{p, q} \rightarrow \Phi^{p-k, q-1}$ для всех $p, q \in \mathbb{Z}_{+}$. 
ПРЕДЛОЖЕНИЕ 5. Для всех $k, l, m, n \in \mathbb{Z}_{+}$справедливы следующие равенства:

1) $\left[\nabla_{l}^{k}, \nabla_{n}^{m}\right]=\sum_{1 \leqslant r \leqslant l}\left[\begin{array}{c}k, m \\ l, n\end{array}\right]_{r} \nabla_{l+n-r}^{k+m-r}$, əде $\left[\begin{array}{c}k, m \\ l, n\end{array}\right]_{r}=\left(\begin{array}{c}m \\ r\end{array}\right)\left(\begin{array}{c}l+n-r \\ n\end{array}\right)-\left(\begin{array}{c}k \\ r\end{array}\right)\left(\begin{array}{c}l+n-r \\ l\end{array}\right)$ $\left(\begin{array}{l}k \\ l\end{array}\right)=k ! /(l !(k-l) !)$

2) $\left[\nabla_{l}^{k}, \lambda^{m}\right]=\left(\begin{array}{c}m \\ l\end{array}\right) \lambda^{k+m-l}$;

3) $\left[\iota_{m}, \nabla_{l}^{k}\right]=\left(\begin{array}{c}m+l-k \\ l\end{array}\right) \iota_{m+l-k}$;

4) $\left\{\lambda^{k}, \lambda^{l}\right\}=0$;

5) $\left\{\lambda^{k}, \iota_{l}\right\}=\delta_{l}^{k} \mathrm{id}_{\Phi}$, әде $\delta_{l}^{k}-$ символ Кронекера;

6) $\left\{\iota_{k}, \iota_{l}\right\}=0$.

Обратим внимание, что здесь $[X, Y]=X \circ Y-Y \circ X-$ коммутатор, $a\{X, Y\}=$ $X \circ Y+Y \circ X-$ антикоммутатор отображений $X, Y: \Phi \rightarrow \Phi$.

Доказательства всех этих формул проводятся прямыми вычислениями. Введем еще на пространстве $\Phi$ следуюшие операции.

- Линейное отображение $\delta: \Phi \rightarrow \Phi$ зададим формулой

$$
\begin{aligned}
& (\delta \phi)\left(\xi_{0}, \ldots, \xi_{q}\right)= \\
& \quad=\frac{1}{q+1} \sum_{0 \leqslant \alpha<\beta \leqslant q}(-1)^{\alpha+\beta-1}\left(\xi_{\alpha}-\xi_{\beta}\right) \phi\left(\xi_{\alpha}+\xi_{\beta}, \xi_{0}, \ldots, \check{\xi_{\alpha}}, \ldots, \check{\xi_{\beta}}, \ldots, \xi_{q}\right)
\end{aligned}
$$

для всех $\phi \in \Phi^{q}, \xi_{0}, \ldots, \xi_{q} \in \mathbb{C}$. В частности, $\delta: \Phi^{p, q} \rightarrow \Phi^{p+1, q+1}$ для всех $p, q \in \mathbb{Z}_{+}$.

- Линейные отображения $\delta_{k}: \Phi \rightarrow \Phi, k \in \mathbb{Z}$, зададим формулой $\delta_{k}=\delta-k \lambda^{1}$. В частности, $\delta_{k}: \Phi^{p, q} \rightarrow \Phi^{p+1, q+1}$ для всех $p, q \in \mathbb{Z}_{+}$.

ПРЕДЛОЖЕНИЕ 6. Справедливы следующие равенства:

1) $\delta \circ \delta=0$;

2) $\left\{\lambda^{i}, \delta\right\}=\lambda^{i+1} \circ \lambda^{0}, i=0,1$;

3) $\left\{\iota_{k}, \delta\right\}=\nabla_{k-1}^{0}-\nabla_{k}^{1}, \quad k \in \mathbb{Z}_{+}$, в частности $\left\{\iota_{0}, \delta\right\}=-\nabla_{0}^{1}, \quad\left\{\iota_{1}, \delta\right\}=\nabla_{0}^{0}-\nabla_{1}^{1}$;

4) $\left[\nabla_{0}^{1}, \delta\right]=0$;

5) $\left\{\lambda^{i}, \delta_{k}\right\}=\lambda^{i+1} \circ \lambda^{0}, i=0,1$, в частности $\lambda^{0} \circ \delta_{k}+\delta_{k+1} \circ \lambda^{0}=0, k \in \mathbb{Z}$;

6) $\delta_{k} \circ \delta_{k}=-k \lambda^{2} \circ \lambda^{0}, k \in \mathbb{Z}$.

Эти равенства доказьваются прямыми вычислениями.

И еше две операции на пространстве $\Phi$.

- Линейные отображения $R_{k}: \Phi \rightarrow \Phi, k \in \mathbb{Z}$, зададим формулой $R_{k}=\nabla_{0}^{0}-\nabla_{1}^{1}-$ $k \mathrm{id}_{\Phi}$. В частности, $R_{k}: \Phi^{p, q} \rightarrow \Phi^{p, q}$, причем $\left.R_{k}\right|_{\Phi^{p, q}}=(q-p-k) \mathrm{id}_{\Phi^{p, q}}$, откуда ker $R_{k} \cap$ $\Phi^{q}=\Phi^{q-k, q}, q \in \mathbb{Z}$.

- Линейные отображения $\rho_{k}:{ }_{k} \Phi \rightarrow{ }_{k} \Phi, k \in \mathbb{Z}$, где

$$
{ }_{k} \Phi=\bigoplus_{q \geqslant 0} \Phi_{\neq(q-k)}^{q},
$$

зададим формулами

$$
\left(\rho_{k} \phi\right)\left(\xi_{1}, \ldots, \xi_{q}\right)=-\int_{0}^{1} t^{k-q-1} \phi\left(t \xi_{1}, \ldots, t \xi_{q}\right) d t
$$


для всех $\phi \in \Phi_{\geqslant p}^{q}, p>q-k, \xi_{1}, \ldots, \xi_{q} \in \mathbb{C}$, и

$$
\left.\rho_{k}\right|_{\Phi^{p, q}}=(q-p-k)^{-1} \mathrm{id}_{\Phi^{p, q}}
$$

для $p \neq q-k$. Легко проверить, что эти формулы согласованы друг с другом и действительно определяют серию линейных отображений на ${ }_{k} \Phi, k \in \mathbb{Z}$.

ПРЕДЛОЖЕнИЕ 7. Определены изоморфизмы линейных локально-выпуклых пространств

$$
R_{k}:{ }_{k} \Phi \simeq{ }_{k} \Phi, \quad\left(R_{k}\right)^{-1}=\rho_{k}, \quad k \in \mathbb{Z} .
$$

Для доказательства достаточно посмотреть на действие введенных отображений на однородных компонентах $\Phi^{p, q}$.

ПРЕДЛОЖЕНИЕ 8. Справедливы следующие равенства:

1) $\lambda^{l} \circ R_{k}=R_{k+1-l} \circ \lambda^{l}, \quad l, k \in \mathbb{Z}$;

2) $\lambda^{l} \circ \rho_{k}=\rho_{k+1-l} \circ \lambda^{l}, \quad l, k \in \mathbb{Z}$;

3) $\left[\delta, R_{k}\right]=0, \quad k \in \mathbb{Z}$

4) $\left[\delta, \rho_{k}\right]=0, \quad k \in \mathbb{Z}$

5) $\left\{\iota_{1}, \delta_{k}\right\}=R_{k}, \quad k \in \mathbb{Z}$.

Доказательства этих равенств сводятся к проверке действий на однородных компонентах с использованием предложений 5 и 6.

2.3. Вспомогательные комплексы. Согласно п. 4,6 предложения 5 линейные отображения $\lambda^{0}, \iota_{0}: \Phi \rightarrow \Phi$ удовлетворяют равенствам $\lambda^{0} \circ \lambda^{0}=\iota_{0} \circ \iota_{0}=0$, так что определены комплексы $\left\{\Phi ; \lambda^{0}\right\},\left\{\Phi ; \iota_{0}\right\}$. Положим $\Psi=\operatorname{ker} \lambda^{0}=\left\{\phi \in \Phi: \lambda^{0} \phi=0\right\}$ и $\Omega=$ $\operatorname{ker} \iota_{0}=\left\{\phi \in \Phi: \iota_{0} \phi=0\right\}$. Гомотопическая формула $\left\{\lambda^{0}, \iota_{0}\right\}=\mathrm{id}_{\Phi}$ (см. п. 5 предложения 5) влечет

ПРЕДЛОЖЕНИЕ 9. Справедливы следующие утверждения:

1) имеет место разложение $\Phi=\Psi \oplus \Omega\left(\phi=\phi_{\Psi}+\phi_{\Omega}\right.$, где $\phi_{\Psi}=\lambda^{0}\left(\iota_{0} \phi\right), \quad \phi_{\Omega}=$ $\left.\iota_{0}\left(\lambda^{0} \phi\right)\right)$

2) комплекс $\left\{\Phi ; \lambda^{0}\right\}$ имеет тривиальнье когомологии;

3) комплекс $\left\{\Phi ; \iota_{0}\right\}$ имеет тривиальные гомологии.

В силу п. 5 предложения $6 \delta_{k}: \Psi^{p, q} \rightarrow \Psi^{p+1, q+1}$, где $\Psi^{p, q}=\Phi^{p, q} \cap \Psi$, для всех $p, q \in$ $\mathbb{Z}_{+}$, а согласно п. 6 предложения $\left.6 \delta_{k} \circ \delta_{k}\right|_{\Psi}=0$, так что определены комплексы $\left\{\Psi ; \delta_{k}\right\}$ для всех $k \in \mathbb{Z}$.

В дополнение к определенным вьше пространствам ${ }_{k} \Phi$ введем пространства ${ }^{k} \Phi=$ $\bigoplus_{q \geqslant 0} \Phi^{q-k, q}$, так что

$$
\Phi={ }^{k} \Phi \oplus_{k} \Phi \quad \text { для каждого } \quad k \in \mathbb{Z} .
$$

Аналогичным образом, положим ${ }_{k} \Psi={ }_{k} \Phi \cap \Psi_{\text {и }}{ }^{k} \Psi={ }^{k} \Phi \cap \Psi$, так что $\Psi={ }^{k} \Psi \oplus_{k} \Psi$ для каждого $k \in \mathbb{Z}$. По построению $\delta_{k}:{ }^{k} \Psi \rightarrow{ }^{k} \Psi$ и $\delta_{k}:{ }_{k} \Psi \rightarrow{ }_{k} \Psi$, следовательно, определены комплексы $\left\{{ }^{k} \Psi ; \delta_{k}\right\}$ и $\left\{k{ }_{k} \Psi ; \delta_{k}\right\}$, причем

$$
\left\{\Psi ; \delta_{k}\right\}=\left\{{ }^{k} \Psi ; \delta_{k}\right\} \oplus\left\{{ }_{k} \Psi ; \delta_{k}\right\} \quad \text { для каждого } k \in \mathbb{Z} .
$$


ЛЕмма 1. Комплексы $\left\{{ }_{k} \Psi ; \delta_{k}\right\}$ имеют тривиальные когомологии, так что когомологиน

$$
H^{q}\left(\left\{\Psi ; \delta_{k}\right\}\right)=H^{q}\left(\left\{{ }^{k} \Psi ; \delta_{k}\right\}\right), \quad q \in \mathbb{Z}_{+}, \quad k \in \mathbb{Z} .
$$

Действительно, в силу предложения 7 и п. 2, 4, 5 предложения 8 на пространствах ${ }_{k} \Psi$ определены гомотопические формулы $\left\{S_{k}, \delta_{k}\right\}=\mathrm{id}$, где $S_{k}=R_{k}$ ○ $\iota_{1}$, что и влечет точность указанных комплексов (заметим, что отображения $R_{k}, \rho_{k}: \Psi \rightarrow \Psi$ в силу п. 1 и 2 предложения 8).

ЛЕмма 2. Линейные пространства ${ }^{k} \Psi=0$ и, в частности, комплексь $\left\{{ }^{k} \Psi ; \delta_{k}\right\}$ нулевье при $k \geqslant 2$.

Действительно, согласно предложению $4 \Phi^{q-k, q}=0$ при $q-k<q(q-1) / 2$, т.е. при $q^{2}-3 q+2 k>0$, а последнее неравенство верно для всех $q \in \mathbb{Z}$, если $k \geqslant 2$.

ЛЕмма 3. Когомологии комплекса $\left\{{ }^{0} \Psi ; \delta_{0}\right\}$ равньи

$$
H^{q}\left(\left\{{ }^{0} \Psi ; \delta_{0}\right\}\right)= \begin{cases}\mathbb{C} \cdot \lambda^{0} \xi^{2}, & q=2, \\ \mathbb{C} \cdot \lambda^{0} \chi, & q=3, \\ 0, & q \neq 2,3,\end{cases}
$$

где $\left(\lambda^{0} \xi^{2}\right)\left(\xi_{1}, \xi_{2}\right)=\left(\xi_{2}^{2}-\xi_{1}^{2}\right) / 2, \quad \chi\left(\xi_{1}, \xi_{2}\right)=3\left(\xi_{2}-\xi_{1}\right) \xi_{1} \xi_{2}$ (заметим, что $\lambda^{0} \chi=V_{3}-$ определитель Вандермонда 3-го порядка).

Действительно, согласно предложениям 4 и $10\left({ }^{0} \Psi\right)^{q}=\Psi^{q, q}=\lambda^{0} \Phi^{q, q-1}=0$ при $q \neq$ 2,3, тогда как $\Phi^{2,1}=\mathbb{C} \cdot \xi^{2}$, а $\Phi^{3,2}=\mathbb{C} \cdot \chi$, причем в силу п. 5 предложения $6 \delta_{0}\left(\lambda^{0} \xi^{2}\right)=$ $-\lambda^{0}\left(\delta_{-1} \xi^{2}\right)=0$, поскольку $\delta_{-1} \xi^{2}=-\lambda^{0} \xi^{3}$.

Лемма 4. Когомологии комплекса $\left\{{ }^{1} \Psi ; \delta_{1}\right\}$ равнь

$$
H^{q}\left(\left\{{ }^{1} \Psi ; \delta_{1}\right\}\right)= \begin{cases}\mathbb{C} \cdot \lambda^{0} 1, & q=1, \\ \mathbb{C} \cdot \lambda^{0} \xi, & q=2, \\ 0, & q \neq 1,2,\end{cases}
$$

где $\left(\lambda^{0} 1\right)(\xi)=1, \quad \lambda^{0} \xi=V_{2} / 2$, где $V_{2}$ - определитель Вандермонда 2-го порядка.

Действительно, в силу предложений 4 и $10\left({ }^{1} \Psi\right)^{q}=\Psi^{q-1, q}=\lambda^{0} \Phi^{q-1, q-1}=0$ при $q \neq 1,2$, тогда как $\Phi^{0,0}=\mathbb{C}$, а $\Phi^{1,1}=\mathbb{C} \cdot \xi$, причем согласно п. 5 предложения $6 \delta_{1}\left(\lambda^{0} 1\right)=$ $-\lambda^{0}\left(\delta_{0} 1\right)=0$, поскольку $\delta_{0} 1=-\lambda^{0} \xi$.

Комбинируя леммы $1-4$, убеждаемся, что справедлива

Теорема 4. Когомологии комплексов $\left\{\Psi ; \delta_{k}\right\}$ равнь

$$
H^{q}\left(\left\{\Psi ; \delta_{k}\right\}\right)=\mathbb{C} \cdot \lambda^{0} Q_{k}^{q}, \quad q, k \in \mathbb{Z}_{+},
$$

әде

$$
Q_{k}^{q}=\delta_{k}^{0}\left(\delta_{2}^{q} \xi^{2}+\delta_{3}^{q} \chi\right)+\delta_{k}^{1}\left(\delta_{1}^{q} 1+\delta_{2}^{q} \xi\right) \in \Omega^{q-1},
$$

$\delta_{k}^{q}-$ символ Кронекера, $\chi\left(\xi_{1}, \xi_{2}\right)=3\left(\xi_{2}-\xi_{1}\right) \xi_{1} \xi_{2}$. 
2.4. Операции в пространстве $\mathcal{F}$. Как и в предыдущем пункте, выделим в пространствах $\mathcal{F}^{q}=\mathcal{E} \widehat{\otimes} \Phi^{q}$ подпространства $\mathcal{F}^{p, q}=\mathcal{E} \widehat{\otimes} \Phi^{p, q}, \mathcal{F}_{\neq p}^{q}=\mathcal{E} \widehat{\otimes} \Phi_{\neq p}^{q}, \mathcal{F}_{\geqslant p}^{q}=\mathcal{E} \widehat{\otimes}$ $\Phi_{\geqslant p}^{q}, p, q \in \mathbb{Z}_{+}$. Отождествление $\phi=1 \otimes \phi, \phi \in \Phi$, дает вложение $\Phi \subset \mathcal{F}$. В свою очередь, отождествление $X=\mathrm{id}_{\mathcal{E}} \otimes X$ распространяет каждое отображение $X$, первоначально определенное на пространстве $\Phi$, на пространство $\mathcal{F}$, так что все операции из п. 2.2 определены на $\mathcal{F}$.

В частности, определены линейные отображения $\lambda^{0}, \iota_{0}: \mathcal{F} \rightarrow \mathcal{F}$, и мы положим $\mathcal{K}=$ $\operatorname{ker} \lambda^{0}=\mathcal{E} \widehat{\otimes} \Psi$ и $\mathcal{M}=\operatorname{ker} \iota_{0}=\mathcal{E} \widehat{\otimes} \Omega$. Очевидно, что все вычисления, приведенные в п. 2.3, останутся справедливыми с заменой $\Psi$ на $\mathcal{K}, \Omega$ на $\mathcal{M}$ и $\mathbb{C}$ на $\mathcal{E}$.

Введем на пространстве $\mathcal{F}$ собственную операцию.

- Линейное отображение $d_{x}: \mathcal{F} \rightarrow \mathcal{F}$ зададим формулой $d_{x}=\partial_{x} \circ \lambda^{0}$, где $\partial_{x}=\partial / \partial x-$ частная производная по $x$. В частности, $d_{x}: \mathcal{F}^{p, q} \rightarrow \mathcal{F}^{p, q+1}$ для всех $p, q \in \mathbb{Z}_{+}$.

ПРЕДЛОЖЕнИЕ 10. Справедливы следующие равенства:

1) $d_{x} \circ d_{x}=0$

2) $d_{x} \circ \delta_{k}+\delta_{k+1} \circ d_{x}=0, \quad k \in \mathbb{Z}$

3) $\left\{d_{x}, \lambda^{k}\right\}=0, k \in \mathbb{Z}_{+}$.

В силу п. 1 предложения 10 определен комплекс $\left\{\mathcal{F} ; d_{x}\right\}$.

ПРЕДЛОЖЕНИЕ 11. Комплекс $\left\{\mathcal{F} ; d_{x}\right\}$ имеет когомологии

$$
H^{q}\left(\left\{\mathcal{F} ; d_{x}\right\}\right)=\Omega^{q}, \quad q \in \mathbb{Z}_{+} .
$$

Действительно, пусть $\phi \in \Omega$, тогда формула $[\phi]=\phi+d_{x} \mathcal{F}$ определяет когомологию, поскольку $d_{x} \phi=\lambda_{0} \phi_{x}^{\prime}=0$, причем $[\phi]=0$, т.е. $\phi=d_{x} g=\lambda^{0} g_{x}^{\prime}$, тогда и только тогда, когда $\phi=0$ в силу п. 1 предложения 9 . С другой стороны, пусть дана когомология $[f]$, где $d_{x} f=\lambda^{0} f_{x}^{\prime}=0$, тогда с помошью предложения 9 проверяется, что $f=\phi+d_{x} g$, где $\phi=\iota_{0}\left(\lambda^{0} f(0)\right) \in \Omega, g=\iota_{0} F, F_{x}^{\prime}=f$ (поясним, что $\left.f(0)(\xi)=f(0 ; \xi), \xi \in \mathbb{C}^{q}\right)$.

\section{3. ДОКАЗАТЕЛЬСТВА ТЕОРЕМ 1-3}

3.1. Доказательство теоремы 1. В силу предложения 1 достаточно вычислить когомологии комплекса $\left\{\Phi ; d_{\mathbb{C}}\right\}$, т.е. комплекса $\left\{\Phi ; \delta_{0}\right\}$, поскольку по определению $d_{\mathbb{C}}=$ $\delta=\delta_{0}$ (заметим, что $\delta_{0} \circ \delta_{0}=\delta \circ \delta=0$, см. п. 1 предложения 6). Вычисления проводятся в духе п. 2.3.

По построению $\Phi={ }^{0} \Phi \oplus_{0} \Phi$, причем $\delta_{0}:{ }^{0} \Phi \rightarrow{ }^{0} \Phi$ и $\delta_{0}:{ }_{0} \Phi \rightarrow{ }_{0} \Phi$, так что имеется разложение в прямую сумму комплексов

$$
\left\{\Phi ; \delta_{0}\right\}=\left\{{ }^{0} \Phi ; \delta_{0}\right\} \oplus\left\{0 \Phi ; \delta_{0}\right\} .
$$

Для комплекса $\left\{{ }_{0} \Phi ; \delta_{0}\right\}$ имеем гомотопическую формулу $\left\{S_{0}, \delta_{0}\right\}=\mathrm{id}$, где $S_{0}=R_{0} \circ \iota_{1}$, так что его когомологии тривиальные и надо вычислить когомологии комплекса $\left\{{ }^{0} \Phi ; \delta_{0}\right\}$. По определению $\left({ }^{0} \Phi\right)^{q}=\Phi^{q, q}, q \in \mathbb{Z}_{+}$. Согласно предложению $4 \Phi^{q, q}=0$ при $q<q(q-$ 1) $/ 2$, т.е. при $q>3$. В свою очередь, простые рассуждения дают $\Phi^{0,0}=\mathbb{C}, \Phi^{1,1}=\mathbb{C} \cdot \xi$, 
$\Phi^{2,2}=\mathbb{C} \cdot \delta \xi, \Phi^{3,3}=\mathbb{C} \cdot V_{3}$, где $V_{3}$ - определитель Вандермонда 3-го порядка. Учитьвая, что $\delta=0$ на $\Phi^{0}=\mathbb{C}$, приходим к выводу, что когомологии $H^{q}\left(\left\{{ }^{0} \Phi ; \delta_{0}\right\}\right)=0$ при $q \neq 0,3$, тогда как $H^{0}\left(\left\{{ }^{0} \Phi ; \delta_{0}\right\}\right)=\mathbb{C}$ и $H^{3}\left(\left\{{ }^{0} \Phi ; \delta_{0}\right\}\right)=\mathbb{C} \cdot V_{3}$, причем, как отмечалось ранее, $L^{-1}\left[V_{3}\right]=W_{3}(0)$. Теорема 1 доказана.

3.2. Обобщающая серия комплексов. Введем в пространстве $\mathcal{F}$ ряд операций.

- Линейные отображения $\Delta_{k}: \mathcal{F} \rightarrow \mathcal{F}, k \in \mathbb{Z}$, зададим формулой $\Delta_{k}=\delta_{k}+\nabla_{0}^{1} \circ \lambda^{0}$.

- Линейные отображения $d_{k}: \mathcal{F} \rightarrow \mathcal{F}, k \in \mathbb{Z}$, зададим формулой $d_{k}=d_{x}+\Delta_{k}$.

ПредлОЖениЕ 12. Для всех $k \in \mathbb{Z}$ справедливы следующие равенства:

1) $\Delta_{k} \circ \Delta_{k}=0$

2) $\delta_{k} \circ \lambda^{0}+\lambda^{0} \circ \Delta_{k}=0$, в частности $\delta_{k} \circ d_{x}+d_{x} \circ \Delta_{k}=0$;

3) $\left\{\iota_{0}, \Delta_{k}\right\}=0$

4) $d_{k} \circ d_{k}=0$.

Доказательства проводятся прямыми вычислениями с использованием формул из п. 2.2 и п. 1 предложения 10.

ЗАмечАниЕ. В более общем виде можно ввести линейные отображения $\Delta_{k m}=\delta-$ $k \lambda^{1}+m \nabla_{0}^{1} \circ \lambda^{0}$ с некоторыми $k, m \in \mathbb{C}$. В этом случае композиция $\Delta_{k m} \circ \Delta_{k m}=0$ тогда и только тогда, когда $m=1, k \in \mathbb{C}$.

Итак, определена серия комплексов $\left\{\mathcal{F} ; d_{k}\right\}, k \in \mathbb{Z}$; вычислим соответствуюшие когомологии.

Лемма 5. Уравнение $\lambda^{0} \circ \Delta_{k} f=0, f \in \mathcal{F}^{q}, k, q \in \mathbb{Z}_{+}$, имеет общее решение

$$
f=a Q_{k}^{q+1}+\lambda^{0} g+\Delta_{k} h, \quad a \in \mathcal{E}, \quad g, h \in \mathcal{F}^{q-1},
$$

функиия $Q_{k}^{q}$ определена в теореме 4.

Действительно, всякая функция указанного вида является решением данного уравнения. С другой стороны, пусть $f \in \mathcal{F}^{q}$ и $\lambda^{0} \circ \Delta_{k} f=0$, тогда согласно п. 2 предложения 12 $\delta_{k}\left(\lambda^{0} f\right)=0$, причем $\lambda^{0} f \in \mathcal{K}^{q+1}$. Отсюда в силу теоремы $4 \lambda^{0} f=a \lambda^{0} Q_{k}^{q+1}+\delta_{k} \tilde{h}$, где $a \in \mathcal{E}, \tilde{h} \in \mathcal{K}^{q}$. Согласно п. 2 предложения $9 \tilde{h}=-\lambda^{0} h$, где $h \in \mathcal{F}^{q-1}$, так что

$$
\lambda^{0}\left(f-\Delta_{k} h-a Q_{k}^{q+1}\right)=0,
$$

откуда в силу п. 2 предложения $9 f=\lambda^{0} g+\Delta_{k} h+a Q_{k}^{q+1}, g \in \mathcal{F}^{q-1}$.

Лемма 6. Уравнение $d_{x} \circ \Delta_{k} f=0, f \in \mathcal{F}^{q}, k, q \in \mathbb{Z}_{+}$, имеет общее решение

$$
f=a Q_{k}^{q+1}+d_{x} g+\Delta_{k} h+\phi, \quad a \in \mathcal{E}, \quad g, h \in \mathcal{F}^{q-1}, \quad \phi \in \Omega^{q},
$$

функиия $Q_{k}^{q}$ определена в теореме 4.

Для доказательства следует слегка модифицировать предыдущие рассуждения. Именно, пусть $f \in \mathcal{F}^{q}$ и $d_{x} \circ \Delta_{k} f=0$, тогда в силу п. 2 предложения $12 \delta_{k}\left(d_{x} f\right)=0$, 
причем $d_{x} f \in \mathcal{K}^{q+1}$. Отсюда согласно теореме $4 d_{x} f=a_{x}^{\prime} \lambda^{0} Q_{k}^{q+1}+\delta_{k} \tilde{h}$ с некоторыми $a \in \mathcal{E}, \tilde{h} \in \mathcal{K}^{q}$. Согласно п. 2 предложения 9 можно считать $\tilde{h}=-\lambda^{0} h_{x}^{\prime}, h \in \mathcal{F}^{q-1}$, откуда

$$
d_{x}\left(f-a Q_{k}^{q+1}-\Delta_{k} h\right)=0,
$$

так что в силу предложения $11 f-a Q_{k}^{q+1}-\Delta_{k} h=d_{x} g+\phi$ с некоторыми $g \in \mathcal{F}^{q-1}$ и $\phi \in \Omega^{q}$.

ЛЕмма 7. Функиия $Q_{k}^{q}$, введенная в теореме 4, обладает свойствами:

1) $\lambda^{0} Q_{k}^{q}=0$ для тех и только тех $k, q \in \mathbb{Z}_{+}$, для которьх $Q_{k}^{q}=0$;

2) $\Delta_{k} Q_{k}^{q}=0$ для всех $k, q \in \mathbb{Z}_{+}$.

Действительно, по построению функция $Q_{k}^{q} \in \Omega^{q-1}$, так что согласно п. 1 предложения $9 \lambda^{0} Q_{k}^{q}=0$ лишь при условии $Q_{k}^{q}=0$. Далее по построению $\delta_{k}\left(\lambda^{0} Q_{k}^{q}\right)=0$, так что в силу п. 2 предложения $12 \lambda^{0}\left(\Delta_{k} Q_{k}^{q}\right)=0$, откуда согласно п. 2 предложения 9 $\Delta_{k} Q_{k}^{q}=\lambda^{0} P$, где $P=\iota_{0}\left(\Delta_{k} Q_{k}^{q}\right)=-\Delta_{k}\left(\iota_{0} Q_{k}^{q}\right)=0$ с учетом п. 3 предложения 12 и п. 1 предложения 9 .

ТЕОРемА 5. Когомологии комплексов $\left\{\mathcal{F} ; d_{k}\right\}, k \in \mathbb{Z}_{+}$, равньи

$$
H^{q}\left(\left\{\mathcal{F} ; d_{k}\right\}\right)=\mathbb{C} \cdot Q_{k}^{q+1}, \quad q \in \mathbb{Z}_{+},
$$

функция $Q_{k}^{q}$ введена в теореме 4 .

Действительно, пусть $f \in \mathcal{F}^{q}$ и $d_{k} f=0$. Тогда $\Delta_{k} f=-d_{x} f=-\lambda^{0} f_{x}^{\prime}$, откуда $\lambda^{0}$ 。 $\Delta_{k} f=0$ в силу п. 4 предложения 5 , так что согласно лемме $5 f=a Q_{k}^{q+1}+\lambda^{0} g+\Delta_{k} h$ с некоторыми $a \in \mathcal{E}$ и $g, h \in \mathcal{F}^{q-1}$, причем

$$
0=d_{k} f=d_{k}\left(a Q_{k}^{q+1}\right)+\Delta_{k}\left(\lambda^{0} g\right)+d_{x}\left(\Delta_{k} h\right) .
$$

Далее, в силу п. 2 леммы 7

$$
d_{k}\left(a Q_{k}^{q+1}\right)=a_{x}^{\prime} \lambda^{0} Q_{k}^{q+1}+a \Delta_{k} Q_{k}^{q+1}=a_{x}^{\prime} \lambda^{0} Q_{k}^{q+1},
$$

тогда как

$$
\Delta_{k}\left(\lambda^{0} g\right)+d_{x}\left(\Delta_{k} h\right)=\delta_{k}\left(\lambda^{0}\left(g-h_{x}^{\prime}\right)\right),
$$

с учетом п. 2 предложения 12. Итак,

$$
a_{x}^{\prime} \lambda^{0} Q_{k}^{q+1}=-\delta_{k}\left(\lambda^{0}\left(g-h_{x}^{\prime}\right)\right),
$$

так что $a_{x}^{\prime}=0$ и $\delta_{k}\left(\lambda^{0}\left(g-h_{x}^{\prime}\right)\right)=0$ в силу теоремы 4 . Следовательно, $a \in \mathbb{C} \subset \mathcal{E}$, тогда как $\lambda^{0} \circ \Delta_{k}\left(g-h_{x}^{\prime}\right)=0$, и согласно лемме 5

$$
g-h_{x}^{\prime}=b_{x}^{\prime} Q_{k}^{q}+\lambda^{0} r+\Delta_{k} s_{x}^{\prime}
$$

с некоторыми $b \in \mathcal{E}$ и $r, s \in \mathcal{F}^{q-2}$. Подставляя $g=h_{x}^{\prime}+b_{x}^{\prime} Q_{k}^{q}+\lambda^{0} r+\Delta_{k} s_{x}^{\prime}$ в найденное ранее представление для $f$, получаем

$$
\begin{aligned}
f & =a Q_{k}^{q+1}+\lambda^{0}\left(h_{x}^{\prime}+b_{x}^{\prime} Q_{k}^{q}+\lambda^{0} r+\Delta_{k} s_{x}^{\prime}\right)+\Delta_{k} h= \\
& =a Q_{k}^{q+1}+d_{k} h+d_{x}\left(b Q_{k}^{q}\right)+d_{x}\left(\Delta_{k} s\right)+\Delta_{k}\left(b Q_{k}^{q}\right)+\Delta_{k}\left(\Delta_{k} s\right)= \\
& =a Q_{k}^{q+1}+d_{k}\left(h+b Q_{k}^{q}+\Delta_{k} s\right),
\end{aligned}
$$

где мы учли п. 2 леммы 7 и п. 1 предложения 12. Таким образом, когомология $[f]=$ $a Q_{k}^{q+1}$ с некоторой комплексной постоянной $a$, что и требовалось доказать. 
3.3. Доказательство теорем 2 и 3. Развитый выше математический аппарат, в частности предложения 2,3 и теорема 5 , сводят доказательство указанных теорем к элементарной проверке равенств $d_{\mathcal{E}}=d_{1}$ и $d_{\mathfrak{A}}=d_{2}$.

\section{4. ОБСУЖДЕНИЕ}

Когомологии алгебры Ли гладких векторных полей на прямой с коэффициентами в тривиальном представлении можно считать, по сушеству, известными [2], [3], хотя конкретных формулировок, по-видимому, не сушествует. В настоящей работе приведена теорема 1 с целью показать ее место в единой схеме и подчеркнуть общую методику доказательств. В случае коэффициентов в естественном и присоединенном представлениях имеются общие результаты, касающиеся когомологий с нетривиальными коэффициентами [7]-[9], из которых, по-видимому, можно получить явные выражения для изучаемых когомологий, однако необходимые для этого усилия заведомо превосходят затраченные здесь на прямые вычисления. В любом случае полезно иметь элементарные доказательства для основных модельных примеров. Кроме того, судя по всему, развитая при этом техника допускает обобщения на реальные ситуации, имеющие и физический интерес.

Благодарности. Работа выполнена при поддержке РФФИ (гранты № 98-01-00640 и 00-15-96073).

\section{Список литературы}

[1] П. Картье. Когомологии алгебр Ли. В сб.: Теория алгебр Ли. Топология групп Ли. Семинар "Софус Ли". М.: ИЛ, 1962. С. 32.

[2] Д. Б. Фукс. Когомологии бесконечномерных алгебр Ли. М.: Наука, 1984.

[3] Б. Л. Фейгин, Д. Б. Фукс. Когомологии групп и алгебр Ли. В сб.: Итоги науки и техники. Современные проблемы математики. Фундаментальные направления. Т. 21. М.: ВИНИТИ, 1988. C. 121.

[4] J. A. de Azcárraga, J. M. Izquierdo, J. C. Pérez Bueno. An introduction to some novel applications of Lie algebra cohomology in mathematics and physics. arXiv:physics/9803046.

[5] И. М. Гельфанд, Д. Б. Фукс. Функц. анализ и его прилож. 1968. Т. 2. № 4. С. 92.

[6] Л. Хёрмандер. Анализ линейных дифференциальных операторов с частными производными. Т. 1. Теория распределений и анализ Фурье. М.: Мир, 1986.

[7] T. Tsujishita. Proc. Japan Acad. A. 1977. V. 53. P. 134.

[8] М. В. Лосик. Функц. анализ и его прилож. 1972. Т. 6. № 4. С. 44.

[9] В. Н. Решетников. ДАН СССР. 1973. Т. 208. № 5. С. 1041.

Поступила в редакцию 15.I.2001 г. 